\title{
Motor Imagery Decoding in the Presence of Distraction Using Graph Sequence Neural Networks
}

This paper was downloaded from TechRxiv (https://www.techrxiv.org).

\section{LICENSE}

CC BY-NC-SA 4.0

SUBMISSION DATE / POSTED DATE

$12-01-2022$ / 18-01-2022

CITATION

Cai, Shengyuan (2022): Motor Imagery Decoding in the Presence of Distraction Using Graph Sequence Neural Networks. TechRxiv. Preprint. https://doi.org/10.36227/techrxiv.18268196.v1

$\mathrm{DOI}$

10.36227/techrxiv.18268196.v1 


\title{
Motor Imagery Decoding in the Presence of
}

\section{Distraction Using Graph Sequence Neural}

\section{Networks}

\author{
Shengyuan Cai,Haoran Li,Qiang Wu Member, IEEE,Ju Liu Senior Member, IEEE, Yu Zhang Senior
}

Member, IEEE

\begin{abstract}
In this study, we propose a graph sequence neural network (GSNN) to accurately decode patterns of motor imagery from electroencephalograms (EEGs) in the presence of distractions. GSNN aims to build subgraphs by exploiting biological topologies among brain regions to capture local and global relationships across characteristic channels. Specifically, we model the similarity between pairwise EEG channels by the adjacency matrix of the graph sequence neural network. In addition, we propose a node domain attention selection network in which the connection and sparsity of the adjacency matrix can be adjusted dynamically according to the EEG signals acquired from different subjects. Extensive experiments on the public Berlin-distraction dataset show that in most experimental settings, our model performs considerably better than the state-of-the-art models. Moreover, comparative experiments indicate that our proposed node domain attention selection network plays a crucial role in improving the sensibility and adaptability of the GSNN model. Finally, in the process of extracting the intermediate results, the relationships between important brain regions and channels were revealed subject to different influences of different subjects in distraction themes.
\end{abstract}

\section{Index Terms}

Attention, Electroencephalogram, Graph Sequence Neural Network, Motor Imagery, Visual and Auditory Distractions

\section{INTRODUCTION}

ELECTROENCEPHALOGRAM (EEG) is an important way for the decoding of brain signals that reflect the dynamic activity of neurons originating in the central nervous system and respond quickly to different brain states [1] . Motor imagery (MI) is a commonly used noninvasive brain-computer interface (BCI) paradigm [2]-[4]. When

This work was supported by the National Key Research and Development Program (Grant No.2020YFC0833204), the Shandong Province Key Innovation Project (Grant No.2020CXGC010903), and the National Academic Innovation Fund of China Education Bureau (Grant No.202110422135). (Corresponding author: Qiang Wu, email: wuqiang@sdu.edu.cn.)

Shengyuan Cai,Haoran Li, Qiang Wu,Ju Liu are with the School of Information Science and Engineering, Shandong University, Qindao China and the Institute of Brain and Brain-Inspired Science, Shandong University, Jinan, China (e-mail: bert.cai.sdu@ gmail.com, wuqiang @ sdu.edu.cn, juliu@sdu.edu.cn).

Yu Zhang is with the Department of Bioengineering, Lehigh University, Bethlehem 18015, USA (e-mail:yuzi20@lehigh.edu). 
a person imagines or pretends body movements, the corresponding MI response is evoked in the brain, and a large number of neuronal activities occur in the motor cortex [5]. People with disabilities can control auxiliary robots [1],[38] or wheelchairs [3],[39] and perform daily activities by implementing MI-based BCI system, which has been shown to contribute to stroke rehabilitation [4],[6],[7]. However, recent studies found that the performance of the MI-based BCI system depends heavily on the behaviors of subjects, such as the case of performing kinesthetic MI as visual MI. According to Friedrichetal et al. [8], passive auditory distraction improves the subjects' performance in MI. By contrast, Alvarsson et al. proposed that both positive and negative auditory interferences can lead to the extension of imagery time, and found that there is a slight difference between positive distraction and negative distraction, thus indicating that different forms of auditory interferences (natural and noisy environments) have different effects on individual well-beings and arousal levels [9]. Studies have examined the differences in brain activation patterns when eyes were open and closed by considering the effects of distraction themes. Even if these studies were conducted under the same conditions, different brain activation patterns could be found in both cases [10]. In the open state of eyes, an increase in the level of awakening and increased eye movements are observed when the attention load increases [11]. Compared with the closed state, the "external sensory" network in the open state, including the attention, eye movement, and arousal systems, are more activated. In contrast, the "endosensory" network, including the visual, auditory, and sensory systems, along with some default mode networks, is more active when eyes are closed than when eyes are open [12]. Therefore, subject to the influence of different distraction paradigms, accurate decoding of MI-associated EEG patterns becomes highly challenging, and thus require more sophisticated algorithms that are robust to noise disturbances caused by distraction.

So far, many traditional machine learning algorithms have been used for MI EEG classification, such as linear discriminant analysis (LDA), random forest (RF), and support vector machine (SVM). However, owing to the low signal-to-noise ratio (SNR) values of the EEG signals, the original EEG data are usually contaminated by noise. Therefore, the distribution of the original EEG samples is chaotic. In particular, this situation is more evident in the case of the MI task in the presence of distraction. To solve this problem, numerous research efforts have been devoted to the development of feature extraction methods to uncover MI-driven EEG features before classification. Common spatial pattern (CSP) [4] is a popular feature extraction method for MI BCI tasks. In the past decade, many CSP variants have been proposed to optimize EEG features for improved MI decoding. For example, Novietal [3] proposed a subband CSP (SBCSP) method, to extract the function of each frequency band of the original EEG signal. However, this algorithm cannot extract the spatial features of the signal. Inspired by the idea of multisubband input, Ang et al. [1] proposed the filter bank common space pattern (FBCSP), which uses bandpass filters and the CSP algorithm to extract optimal spatial features, while maintaining high-subject sensitivity. Although these approaches showed strengths in improving MI decoding, all of them have been studied under a distraction- free experimental paradigm, wherein the SNR of the EEG was guaranteed by the full engagement of subjects. However, in natural and complex environments with various distractions, use of these traditional algorithms for EEG-based MI decoding may face the following challenges:

1.Given that subjects are affected differently by the same independent distraction theme, the EEG signals of different subjects vary greatly, which hinders the generality of classifiers in specific distraction themes 
2.The topological structure of EEG channels may not be effectively utilized to learn specific EEG representations

3.Participants may not always produce the expected imagery under certain distraction themes [13]. Consequently, the distribution of the original EEG in the collected data may be noisy, thus making it impossible to extract valid features

To overcome these problems, researchers have proposed many end-to-end MI EEG decoding methods based on deep learning techniques [36]. For example, Li et al. [14] proposed an end-to-end framework called channel projection mixed scale convolution neural network (CPMixedNet) to improve the performance of MI EEG decoding. Long- and short-term memory (LSTM) model is suitable for time series feature learning, although it ignores spatial information that is important for neural pattern decoding [16]. Wang et al. [15] proposed long- and short-term memory based eeg classification (Em-LSTM) to solve the problem of gradient disappearance or gradient explosion in the training process of convolution neural networks. Generally, the method based on deep learning can achieve better performance than traditional methods because of the advantages provided by learning and embedding of feature separation and classification in a single network. However, existing EEG coding methods based on deep learning do not consider the graph structure of EEG signals. Based on the convolution neural network, the convolution operation on a graph can be defined in the spectral and nonspectral domains. The spectral method based on the redefinition of the Fourier convolution operation on the graph can effectively extract feature information, including topological structures information in MI-BCI tasks. Kipf et al. [17] proposed a hierarchical propagation rule, which uses the Chebyshev expansion method to simplify the approximation of the graph Laplacian [18]. The graphical attention network [19] may be used to calculate the representation of nodes in an entire community based on the attention mechanism [20]. Wu et al. proposed a active framework named Simplifying Graph Convolutional Networks (SGC) [40]. These methods improve the performance of graph-related tasks. Additionally, for graph structures, the real characteristics of low frequency have sufficient information for machine learning tasks [21]. Simultaneously, unsupervised domain adaptation aims to alleviate the domain transfer from the supervised source to the unsupervised target domains in the process of knowledge transfer. The domain-invariant feature learning method may be used to align the features of the source and target domains to the common feature space. This alignment can be achieved by minimizing bifurcation [22] or maximizing reconstruction [23], or by adversarial training [24]. The node domain adaptation (NodeDAT) regularizer proposed by Miao et al. [25] extends domain adversarial training into graph neural networks. By minimizing the difference between the features in the source and target domains of each node, NodeDAT achieves finer-grained regularization. In this study, we propose a novel graph sequence neural network combined with a nodal domain attention selection network for the MI task subject to the influences of various distractions. With nodal domain confrontation training, our proposed model achieves a stronger generalization ability and can effectively solve the problems of BCI blindness and poor goal realization of MI subjects. The primary contributions of this study are as follows:

1.A preprocessing regularization map algorithm is proposed, which is suitable for preprocessing EEG signals in the presence of distraction and improves the robustness of the model against noisy data

2.A gated graph convolutional network based on EEG signals is proposed, which can effectively capture the essential patterns of MI subjects in distraction and improve the classification accuracy 


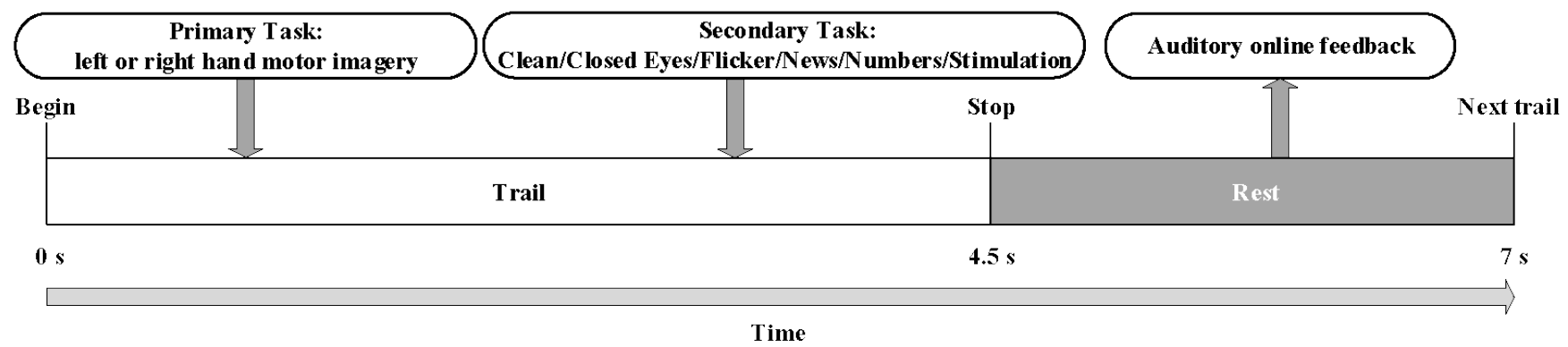

Fig. 1. Sequence of task paradigms. The subjects performed left- and right-hand motor movements subject to the imagery influences of different secondary tasks (none, clean, eye closure, flicker, news, and number).

3. A node domain adversarial training method for different independent distraction themes is proposed to improve the self-adaptability of the network

The remainder of this paper is organized as follows. In section 2, we introduce the dataset and the baseline methods commonly used for graph representation learning and our designed GSNN network. Section 3 presents the experimental results of the GSNN. On Berlin Institute of Technology public datasets, our proposed model was validated with extensive experiments in comparison with benchmark models. Section 4 discusses some detail segments and potential applications

\section{MATERIALS AND METHODS}

\section{A. Dataset}

A public dataset collected by the Technical University of Berlin from 16 healthy subjects between the ages of 24 and 28 was used for our study [26]. These patients were assigned to exercise imagery experiments in the presence of different independent distraction themes. For each subject, the main experiment was divided into seven runs for $10 \mathrm{~min}$, and 72 secondary experiments were run each time; one experiment lasted $4.5 \mathrm{~s}$. The subjects were asked to choose a tactile hand movement subject to the influence of different secondary tasks (clean, eye closure, news, numbers, flicker, stimulation). There are several MI strategies for subjects to choose from. For example, squeezing a soft ball, turning on the faucet, playing the piano, and using a saltshaker. The experiment used the Fast'nEasyCap (EasyCapGmbH) system, which consisted of 63 wet $\mathrm{Ag} / \mathrm{AgCl}$ electrodes placed in a symmetrical position according to the International 10-20 system [27], with reference to the nose. Moreover, two 32-channel amplifiers were used to amplify the signal at a sampling rate of $100 \mathrm{~Hz}$. The original EEG signal of the subjects was recorded in detail in the dataset and was applied to the main task tag of each subject to record whether the task was left-handed or right-handed. This study was conducted according to the declaration of Helsinki and was approved by the Ethics Committee of the Charite-Universitätsmedizin Berlin (approval number: EA4/012/12). 
To cross-compare outcomes with other models, we adopted the same preprocessing method as the original dataset, sampled the EEG data at $100 \mathrm{~Hz}$, calculated the Laplace filter of $\mathrm{C} 3$ and $\mathrm{C} 4$, and used the fifth-order Butterworth filter to filter the data in the range of $9-13$ and $18-26 \mathrm{~Hz}[26]$.

\section{B. Graph Filter Neural Network}

We consider a graph $\mathcal{G}=(\mathcal{X}, \mathcal{A})$, where $\mathcal{X}$ denotes a set of nodes and $\mathcal{A}$ denotes a set of edges between nodes in $\mathcal{X}$. If the graph convolution network (GCN) formula [17] is used, the form of score $G \in \mathbb{R}^{N \times 1}$ is as follows.

$$
G=\sigma\left(D^{-\frac{1}{2}} A D^{-\frac{1}{2}} X \Theta_{a t t}\right)
$$

where $\sigma$ is the activation function (e.g. sigmoid), $A \in \mathbb{R}^{N \times N}$ is the adjacency matrix with self-connections, which can represented the edge set $\mathcal{A}$. $D \in \mathbb{R}^{N \times N}$ is the degree matrix of $A, X \in \mathbb{R}^{N \times F}$ is the input features of the graph with $N$ nodes and $F$-dimensional features, which can represent data on $\mathcal{X} . \Theta_{\text {att }} \in \mathbb{R}^{F \times 1}$ is the only parameter of the convolution-layer. In general, GCNs learn a feature transformation function for $X$ and produces the output

$G \in \mathbb{R}^{N \times F^{\prime}}$, where $F^{\prime}$ denotes the output feature dimension. However, when the noise is so large that GCN cannot be reduced by the first-order low-pass filter, it has a risk of overfitting to the noise. In particular, the inner weight $W_{1}$ and $A$ may be trained using high noisy features.

According to Hoang et al. [21], graph filter neural network (gfNN) is a faster structure compared with GCN after the correction of the convolution layer, and the form of multilayer (two-layer) perceptrons is as follows,

$$
G_{g f N N}=h_{\mathrm{MLP}}\left(X \mid W_{1}, W_{2}\right)=\sigma_{2}\left(\sigma_{1}\left(X W_{1}\right) W_{2}\right)
$$

where $\sigma_{1}$ is the entry-wise rectifier linear unit function, and $\sigma_{2}$ is the softmax function. Note that both $\sigma_{1}$ and $\sigma_{2}$ are contraction maps. Additional details are provided in [21].

\section{Self-attention pooling}

Pooling layer enables the GCN model to lessen the number of parameters by reducing the scale of representation, thus avoiding overfitting. According to Zhang et al. [28], SortPool sorts the embedding of nodes and feeds the sorted embedding to the next layer. Conversely, Ying et al. [29] proposed DiffPool, which is a hierarchical graph pooling method for end-to-end learning of allocation matrices.

Inspired by these studies, self-attention pooling is divided in two parts: global and hierarchical pooling methods. The global pooling method considers the graphic function and uses neural networks to aggregate all representations of nodes in each layer. Diagrams with different structures can be processed because the global pool method collects all representations. The hierarchical pooling method is used to construct a model with node assignments based on features or topologies that can be learnt in each layer. $P^{(l)} \in \mathbb{R}^{N_{l} \times N_{l+1}}$ contains the probability values of nodes in layer $l$, which is assigned to clusters in the next layer $l+1$. $A$ denotes an assignment matrix which updates in layer $l+1$ based on $P^{(l)}$. $N_{l}$ denotes the number of nodes in layer $l$. Specifically, the form of assigned nodes is as follows: 


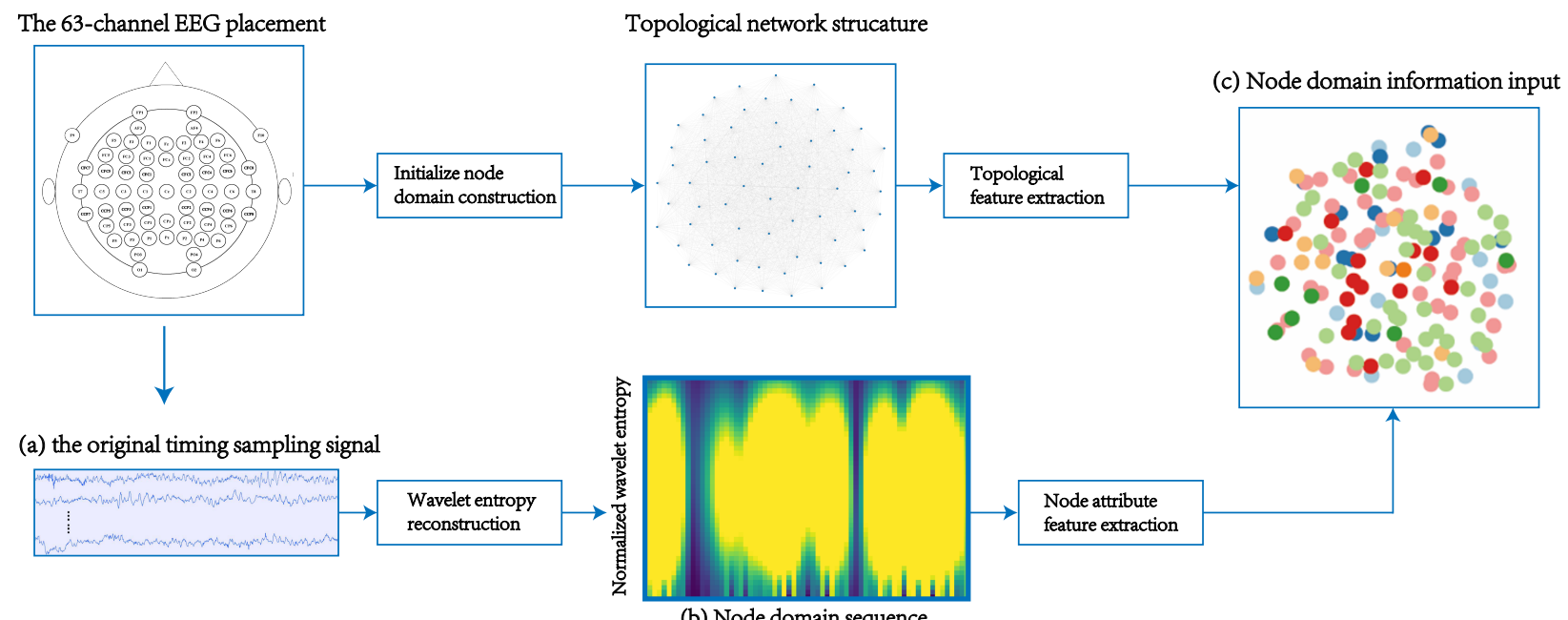

(b) Node domain sequence

Fig. 2. Schematic showing the working principle of lead-to-graph processing conversion. (a).Baseline correction and bandpass filtering processing of the original signal. (b) Extraction of node features in wavelet domain. (c) Construction of a feature map framework.

$$
\begin{gathered}
P^{(l)}=\operatorname{softmax}\left(\operatorname{gfNN}_{l}\left(A^{(l)}, X^{(l)}\right)\right) \\
A^{(l+1)}=P^{(l) \top} A^{(l)} P^{(l)}
\end{gathered}
$$

where $X$ denotes the node feature matrix. The output probability of class $\mathbf{Y}_{i}$ can be computed based on passing each feature matrix $X_{i}$ into model.The form is as follows

$$
p\left(\mathbf{Y}_{i} \mid X_{i}, \theta\right)=\operatorname{softmax}_{\mathbf{Y}_{i}}\left(\operatorname{Com}-\operatorname{pool}\left(\sigma\left(G_{i}\right)\right) W^{O}\right)
$$

where Com-pool denotes the combined pooling across all nodes on the graph, $\mathbf{Y}_{i} \in\{0,1, \ldots, L-1\}$ denotes the label class index, and $L$ denotes the number of classes, and $W^{O} \in \mathbb{R}^{d^{\prime} \times L}$ denotes the output weight matrix.

\section{Node domain adversarial training}

When participants conduct the main task of prompting, owing to the influence of distraction, they may not always produce the expected MI, which may have a negative impact on the performance of the model [27]. Miao et al. [25] introduced the nodal domain distributed learning method (NodeDAT) to learn the distribution of classes, which can solve the influence of different subjects to a certain extent. Specifically, the model converts each training label $\mathbf{Y}_{i} \in\{0,1, \ldots, L-1\}$ into a prior probability distribution of all classes $\hat{\mathbf{Y}}_{i} \in \mathbb{R}^{L}$, where $\hat{\mathbf{Y}}_{i L}$ denotes the probability of class $\mathrm{L}$ in $\hat{\mathbf{Y}}_{i}$. We have migrated this method to make it suitable for the task of motor imagery under distraction. Label has two classes: left and right class indices 0, 1,respectively. We convert $\mathbf{Y}$ as follows

$$
\hat{\mathbf{Y}}_{i}= \begin{cases}\left(1-\frac{1 \epsilon}{2}, \frac{\epsilon}{2}\right), & \mathbf{Y}_{i}=0 \\ \left(\frac{\epsilon}{2}, 1-\frac{1 \epsilon}{2},\right), & \mathbf{Y}_{i}=1\end{cases}
$$


Subsequently, the model establishes two domains, which are labeled as source/training data $X^{O} \in \mathbb{R}^{B \times N \times F}$ (in this subsection, we denote $X$ by $X^{O}$ for better clarity) and unlabelled target/testing data $X^{E} \in \mathbb{R}^{B \times N \times F}$, where in practice $X^{E}$ can be either oversampled or downsampled to have the same number of samples as $X^{O}$ [7], $B$ denotes the number of training samples. The domain classifier aims to minimize the sum of the two binary cross-entropy losses,

$$
\Phi_{D}=-\sum_{i=1}^{B} \sum_{j=1}^{N}\left(\log \left(p_{j}\left(0 \mid X_{i}^{O}, \theta_{D}\right)\right)+\log \left(p_{j}\left(1 \mid X_{i}^{E}, \theta_{D}\right)\right)\right),
$$

where 0 and 1 denote source and target domains respectively, $\theta_{D}$ denotes the parameters of the domain classifier. Intuitively, the domain classifier learns to classify source data as 0 and target data as $1 . O$ is the labelled target, $E$ is the unlabelled target. $p_{j}$ is the domain probability of the $j t h$ node calculated by Ep (5). To make the domain classifier less sensitive to noise input in the early stage of the training process. Miao et al. implemented gradient reversal layer (GRL) in domain training. With the training, the scaling factor $\beta$ gradually increases from 0 to 1 , thus further scaling the inverse gradient. Additional details are provided in [25].

\section{E. Our method: Graph Sequence Neural Network}

In this study, we propose a novel framework, known as the graphic sequence neural network (GSNN), which combines the graph convolution network with self-attention mechanism and node domain adversarial, to predict the downstream task results of MI. We use the notch filter to filter power frequency interference [26], and use wavelet entropy reconstruction as the nodal attribute feature. The dynamic distance ratio is reconstructed as the node connection parameter to construct the adjacency matrix of the edge connection.

1) Node attribute characteristics: We reconstruct the signal based on the time-domain sequence signal after preprocessing. According to Rosso et al. [30], wavelet entropy can measure the degree of order and disorder of the signal and provide potential dynamic process information related to the signal. It selects the appropriate wavelet basis based on the wavelet transform with multiple scales and directions and expands the original signal at different scales [16]. Therefore, we used wavelet entropy reconstruction in each lead as the nodal attribute feature of the EEG channel. The relative wavelet energy of $j t h$ signal at each scale is defined as follows:

$$
p_{h j}=\frac{E_{h}}{E_{\text {total }}}=\frac{\sum_{k=1}^{L_{h}}\left|C_{h}(k)\right|^{2}}{\sum_{h} \sum_{k=1}^{L_{h}}\left|C_{h}(k)\right|^{2}}
$$

Where $C_{h}(k)$ is the decomposition coefficient of each scale $h, L_{h}$ denotes the number of coefficients at each decomposition scale, $E_{h}$ is the wavelet energy, and $E_{\text {total }}$ is the total energy. The wavelet entropy is calculated by the following formula:

$$
X_{j}=-p_{h j} \log \left(p_{h j}\right)
$$

where $X_{j} \in \mathbb{R}^{1 \times 128}$ is one of the element of $X \in \mathbb{R}^{N \times F}$, which is the input features of the graph with 63 nodes and 128-dimensional features. Additional details are provided in [16]. 
2) Node topology reconfiguration: The topological structure of EEG channels is necessary for graphic representation learning. The adjacency matrix $A$ represents the topological pattern, where $f$ represents the number of channels in EEG signals. The weight between channels $m$ and $n$ is indicated by each learnable entry $A_{m n}$. According to Salador et al. [31], the strength of connections between brain regions attenuates as an inverse square function of physical distance. Therefore, we initialized the relationship between local channels in the adjacency matrix as follows,

$$
A_{m n}=\min \left(1, \frac{\delta}{q_{m n}^{2}}\right),
$$

where $\delta>0$ denotes a calibration constant, $q_{m n}, m, n=1,2, \ldots, f$, denotes the physical distance between channels $m$ and $n$, which is computed from their three-dimensional coordinates obtained from the data sheet of the recording device. Achard et al. [32] observed that sparse functional magnetic resonance imaging networks ( $20 \%$ of all possible connections) can typically maximize the efficiency of network topology. Therefore, Setting $\delta$ to 5 implies that $\sim 20 \%$ of the entries in $A$ are non-negligible. Finally, to reduce overfitting, we set $A_{m n}$ as a symmetric matrix.

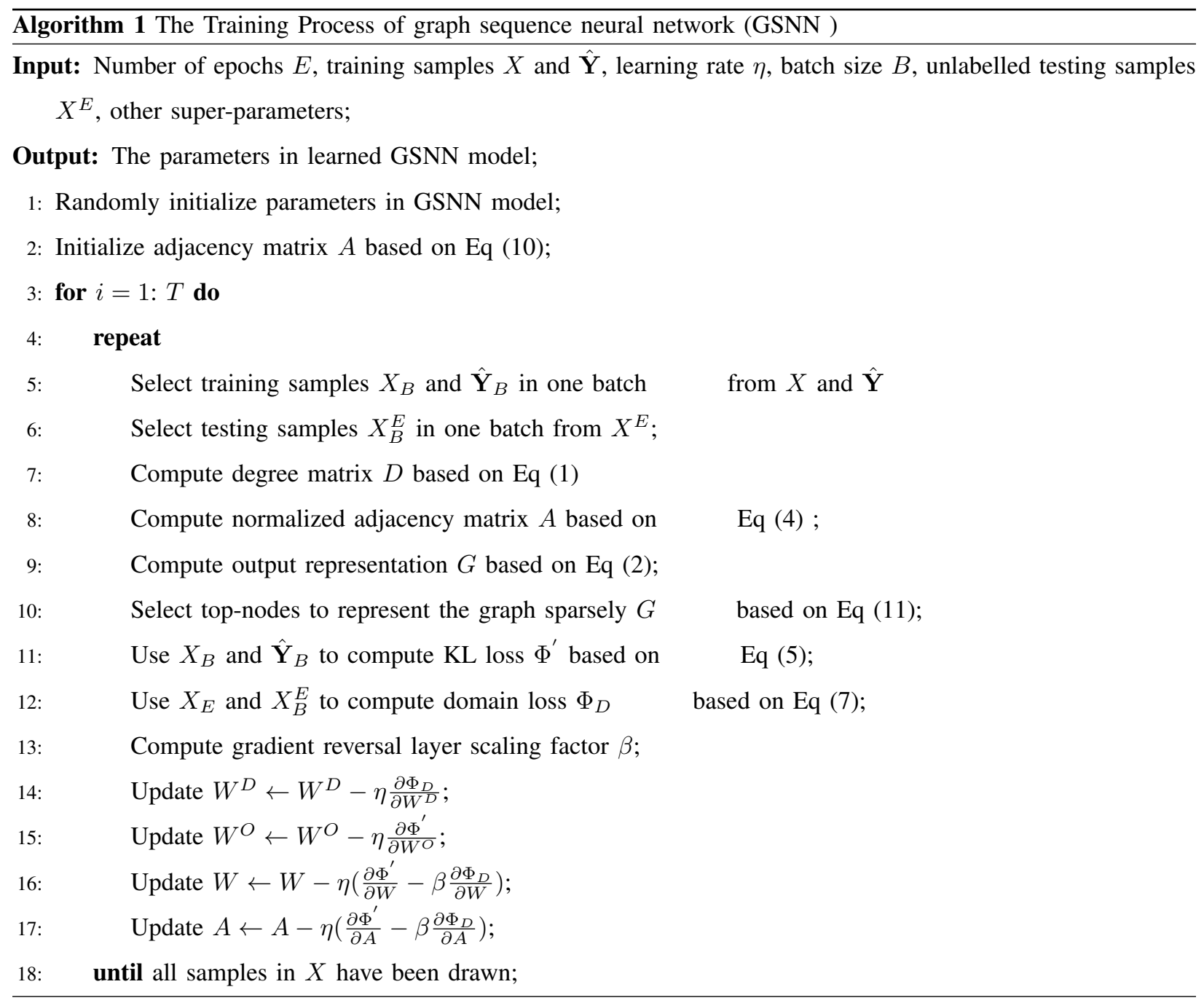


3) Dynamics of GSNN: The characteristics of the reconstructed EEG data are used to train the GSNN network. gfNN realizes the denoising of node features in a high-dimensional space. After 63-dimensional gfNN convolution, the self-attention parameter $G$ of nodal selection is obtained. According to the nodal selection method of [33], even when inputting graphs of different sizes and structures, part of the nodes of the input graph are retained. Therefore, we used self-attention pooling structures to preserve graphic features and topologies, and Top $[k N]$ nodes were selected based on the value of $G$. The pool ratio $k \in(0,1]$ is a superparameter used to determine the number of nodes to be retained.

$$
i d x=\operatorname{top}-\operatorname{rank}(G,[k N]), G_{m a s k}=G_{i d x}
$$

where $i d x$ is an indexing operation and $G_{m a s k}$ is the feature attention mask. The listing top-rank is the function that returns the indices of the top $[k N]$ values. Subsequently, the global pooling and hierarchical merging were executed, respectively, and the label output of the sparse node domain was used as the input of nodeDAT. Because label smoothing mutually mixs uniform noise into other classes, GSNN incorporates more prior knowledge when training with smooth labeling. Through the regularization of the smooth node label, the embedding is conducted by using 63 nodes, and the subsequent round is executed after supervised evaluation. The optimization of the model is determined by the following loss function, including graph network and nodeDAT.

$$
\Phi^{\prime}=\sum_{i=1}^{B} \operatorname{KL}\left(p\left(\mathbf{Y} \mid X_{i}, \theta\right), \hat{\mathbf{Y}}_{i}\right)+\alpha\|A\|_{1},
$$

where $\theta$ denotes the model parameters that need to be optimized, and $\alpha$ denotes the weight of L1 sparse regularization for adjacency matrix $A \cdot p\left(\mathbf{Y} \mid X_{i}, \theta\right)$ denotes the output probability distribution computed via Eq (5). By combining both NodeDAT and graph sequence network, the overall loss function $\Phi^{\prime \prime}$ of GSNN is as follows,

$$
\Phi^{\prime \prime}=\Phi^{\prime}+\Phi_{D}
$$

The detailed algorithm for training GSNN is presented in Algorithm 1. The entire neural network is fine-tuned after GSNN training.

\section{EXPERIMENTAL RESULTS}

\section{A. Experiment Protocols}

According to Glorot et al.[34], even if the evaluation is conducted on the same database, different evaluation schemes can lead to significant differences in results. Generally, the influence of verification methods on test results proceeds as follows: (1) compared with theme-independent evaluation methods, subject-independent evaluation methods are more objective because subjects rely on evaluation methods that disregard individual differences. (2) Subject-level k-fold cross- validation (CV) can evaluate better the sensitivity of the model among different subjects. (3) Independent distraction theme-level k-fold CV can evaluate better the adaptive adjustment ability of the model at different secondary tasks.

Therefore, to avoid data leakage in the evaluation process, we conducted five-fold CV topic independent evaluation protocol under the independent distraction theme of the database. The training and test data used to evaluate the individual difference sensitivity of the model were produced from the same subjects, and there is no information 
TABLE I

Motor Imagery Performance Comparison with DifFerent Network Configurations using all IndePendent Distraction THEMES ON THE BERLIN-MOTOR IMAGERY DATABASE (EM-LSTM: LONG- AND SHORT-TERM MEMORY BASED EEG CLASSIFICATION; BCNN: BINARY CONVOLUTIONAL NEURAL NETWORK)

\begin{tabular}{lccccccc}
\hline \multicolumn{1}{c}{ Model } & calibration & clean & eyes & flicker & news & numbers & stimulation \\
\hline \hline BCNN [36] & 0.5616 & 0.5423 & 0.5304 & 0.5783 & 0.5351 & 0.4694 & 0.5235 \\
Em-LSTM [15] & 0.5335 & 0.5512 & 0.5268 & 0.5536 & 0.5695 & 0.4826 & 0.5099 \\
FBCSP [1] & 0.7016 & 0.8111 & 0.6914 & 0.7222 & 0.7317 & 0.5833 & 0.6139 \\
CPMixedNet [14] & 0.7180 & 0.7737 & 0.7174 & 0.7087 & 0.7240 & 0.7013 & 0.6972 \\
GSNN(Our model) & $\mathbf{0 . 7 7 7 9}$ & $\mathbf{0 . 7 8 1 3}$ & $\mathbf{0 . 7 9 3 5}$ & $\mathbf{0 . 8 2 3 7}$ & $\mathbf{0 . 7 6 9 7}$ & $\mathbf{0 . 7 9 5 9}$ & $\mathbf{0 . 7 8 3 2}$ \\
\hline
\end{tabular}

\begin{tabular}{lccccccc}
\hline \multicolumn{1}{c}{ Model } & \multicolumn{7}{c}{ F-score } \\
\hline \hline calibration & clean & eyes & flicker & news & numbers & stimulation \\
\hline ECNN [36] & 0.5624 & 0.5432 & 0.5321 & 0.5803 & 0.5364 & 0.4777 & 0.5294 \\
FBCSP [1] & 0.5320 & 0.5485 & 0.5251 & 0.5492 & 0.5566 & 0.4626 & 0.4985 \\
CPMixedNet [14] & 0.6908 & 0.7913 & 0.6981 & 0.7113 & 0.7406 & 0.5914 & 0.5830 \\
GSNN(Our model) & $\mathbf{0 . 7 9 2 9}$ & $\mathbf{0 . 7 8 2 5}$ & $\mathbf{0 . 7 9 5 4}$ & $\mathbf{0 . 8 2 6 8}$ & $\mathbf{0 . 7 6 4 7}$ & $\mathbf{0 . 7 9 5 9}$ & $\mathbf{0 . 7 8 1 3}$ \\
\hline & & & & & & & \\
\hline \multicolumn{1}{c}{ Model } & calibration & clean & eyes & flicker & news & numbers & stimulation \\
\hline \hline BCNN [36] & 0.5621 & 0.5429 & 0.5316 & 0.5799 & 0.5363 & 0.4779 & 0.5274 \\
Em-LSTM [15] & 0.5334 & 0.5507 & 0.5263 & 0.5521 & 0.5642 & 0.5021 & 0.5092 \\
FBCSP [1] & 0.6814 & 0.7907 & 0.6873 & 0.7129 & 0.7374 & 0.5907 & 0.5885 \\
CPMixedNet [14] & 0.7031 & 0.7737 & 0.7254 & 0.7055 & 0.7291 & 0.6966 & 0.6986 \\
GSNN(Our model) & $\mathbf{0 . 7 8 3 5}$ & $\mathbf{0 . 7 9 1 9}$ & $\mathbf{0 . 7 9 7 9}$ & $\mathbf{0 . 8 2 4 3}$ & $\mathbf{0 . 7 6 1 6}$ & $\mathbf{0 . 7 9 3 4}$ & $\mathbf{0 . 7 8 3 2}$ \\
\hline
\end{tabular}

overlap between the subjects. Specifically, at each independent distraction theme, we cross-validated 16 subjects at five-fold $\mathrm{CV}$, and repeated the verification process until each subject was treated as a test dataset. In other words, for each independent distraction theme, we repeated it 16 times and calculated the final CV performance as the average of all the test results obtained. This verification method provides a fair evaluation of the performance of the cross-object model and can accurately estimate the possible recognition accuracy of new data from new objects. To compare findings with the results of previous studies, we used four indicators, namely accuracy $\left(P_{a c c}\right)$, recall $\left(P_{r e}\right)$, precision $\left(P_{p r e}\right)$, and F1-score $\left(P_{f}\right)$, to evaluate the performance of the model. The F1-score was introduced as a comprehensive index to balance the influence of accuracy and recall and to evaluate the classifier in a more comprehensive manner. 


\section{B. Network Training}

In the process of training, according to Shchur et al. [35], different data segmentations affect the model performance of graph neural network (GNN). In our experiment, the weight parameters in the con- volution layer were initialized with 20 random seeds and five-fold CV. In a round of cross-validation in the distraction database, samples from 10 secondary experiments were randomly used as test data (a total of 10 secondary tasks $\times 63$ nodes $\times 1953$ edges), and samples from other 62 secondary experiments were used as training data (62 secondary tasks $\times 63$ nodes $\times 3969$ edges). To reduce the time complexity of the computation, the momentum of the ADAM optimizer was 0.9. If the verification loss was not improved after 50 epoch intervals under the epoch termination condition (up to $100 \mathrm{k}$ epochs), we stopped training. The model weight that generated the lowest loss of the validation set was saved as the final parameter. A small batch random gradient descent method was adopted with a fixed learning generator rate of 0.001 and a discriminator of 0.0002. Herein, the small batch size was equal to 10. All models were trained on NVIDIA GeForce RTX 2080 graphics processing unit (GPU), and CUDA 11.0 used Pytorch API.

\section{Evaluation on Database}

TABLE II

PERFORMANCES OF SEQUENCE NEURAL NETWORK AT INDEPENDENT CONDITIONS

\begin{tabular}{cccccccc}
\hline & \multicolumn{7}{c}{ Accuracy-GSNN } \\
\hline Subjects & Calibration & Clean & Eyes & Flicker & News & Numbers & Stimulation \\
\hline \hline P1 & 0.8105 & 0.8067 & 0.7524 & 0.8505 & 0.8362 & 0.8924 & 0.8505 \\
P2 & 0.6933 & 0.5381 & 0.6924 & 0.7486 & 0.6210 & 0.6648 & 0.7781 \\
P3 & 0.8505 & 0.7810 & 0.7638 & 0.8657 & 0.6800 & 0.7952 & 0.8076 \\
P4 & 0.7190 & 0.8352 & 0.8343 & 0.7781 & 0.8143 & 0.8638 & 0.9190 \\
P5 & 0.7543 & 0.8067 & 0.7819 & 0.8495 & 0.9048 & 0.8371 & 0.8210 \\
P6 & 0.8190 & 0.7143 & 0.7781 & 0.8905 & 0.8076 & 0.7524 & 0.7714 \\
P7 & 0.7648 & 0.8190 & 0.8495 & 0.8457 & 0.8067 & 0.7648 & 0.7352 \\
P8 & 0.7924 & 0.8495 & 0.8286 & 0.6943 & 0.6952 & 0.8229 & 0.6486 \\
P9 & 0.8000 & 0.7514 & 0.7514 & 0.7638 & 0.7771 & 0.7143 & 0.6771 \\
P10 & 0.7371 & 0.7781 & 0.7971 & 0.8924 & 0.7352 & 0.8914 & 0.8476 \\
P11 & 0.7505 & 0.8648 & 0.7914 & 0.8362 & 0.5371 & 0.8210 & 0.8771 \\
P12 & 0.8210 & 0.7648 & 0.8781 & 0.7924 & 0.7219 & 0.8352 & 0.6933 \\
P13 & 0.7505 & 0.8219 & 0.7067 & 0.7829 & 0.6771 & 0.7505 & 0.7495 \\
P14 & 0.8486 & 0.8781 & 0.8648 & 0.8076 & 0.9048 & 0.7505 & 0.7905 \\
P15 & 0.7524 & 0.7819 & 0.8629 & 0.8467 & 0.9333 & 0.8352 & 0.7219 \\
P16 & 0.8924 & 0.7790 & 0.8229 & 0.8648 & 0.8229 & 0.6933 & 0.8219 \\
\hline
\end{tabular}

Table I presents a comparison of the results of the proposed method with those of the previous studies. The corresponding verification methods are elucidated. The experimental results show that, compared with other supervised methods, the self-attention framework (based on GSNN) yields a better performance of MI recognition. For the supervised method proposed in [14], the initial evaluation scheme is based on the CV of the subjects 
under all independent distraction themes, and the performance of the cross-subjects is separated. There is a fair comparison between our proposed model and other methods. Based on [14], we used five-fold CV to evaluate their work conducted at each distraction theme (the same method we proposed) and report the results in Table II. The compared results show that the proposed method is better than that reported by Wang et al. [15] In the case of the theme "calibration", the valence of Recall increases from $70.31 \%$ to $78.35 \%$,Precision from $71.80 \%$ to $77.79 \%$, F-score from $71.36 \%$ to $79.29 \%$; In the case of the theme "clean", the valence of Recall increases from $77.37 \%$ to $79.19 \%$,Precision from $77.37 \%$ to $78.13 \%$, F-score from $77.50 \%$ to $78.25 \%$. In the cases of the remaining six distraction themes, the accuracy of the model was improved to a certain extent. The average value of Recall, Precision, and F-score were $80.41 \%, 81.01 \%$ and $80.49 \%$. Figure 4 shows the accuracy assessment of our model and other algorithms. The average growth rates of $P_{a c c}$ and $P_{f}$ were $8.35 \%$ and $14.09 \%$, respectively. The results also showed that, compared with the manual features used in [4], the deep topology and nodal features characterized by GSNN were less sensitive to individual differences.

\section{Evaluation on Different Subjects}

Table II shows the classification effect of the GSNN model for different subjects. On the basis of 16 subjects participating in each distraction experiment, we conducted independent model training and evaluation for each subject. It can be seen that in subjects $1,3,6,12,14$ and 16, the model has good classification accuracy under each distraction theme. In the rest of the subjects, it also showed good performance.

\section{DISCUSSION}

In this section, we study the ablation and analyze the sensitivity of the GSNN model based on the distraction

effect. The relationship between the important brain regions and MI channels is analyzed. Finally, we determine the potential problems of GSNN.

\section{A. Sensitivity Analysis}

To evaluate the sensitivity of the proposed GSNN method proposed, we conducted experiments on datasets with different data enhancement methods. First, we conducted a controlled experiment with no data extension for each distraction topic. Subsequently, considering the same conditions, after the nodal domain adversarial enhancement method, the standard deviation of each independent motion imagery task was calculated. As shown in Table III, compared with other data enhancement methods, the proposed data enhancement method achieves a lower standard deviation. This indicates that the proposed data enhancement method can alleviate overfitting to a great extent and help achieve improved and more stable performances than other methods.

\section{B. Experiments Using Different Structures of GSNN}

By constructing convolution kernels with different types, it can be found that gfNN is more suitable for the classification of motor imagery tasks under independent distraction than other convolution kernels. The accuracy is shown in Table IV, where it can be seen that higher performance can be achieved when gfNN blocks is applied. 
TABLE III

SENSITIVITY OF MOTOR IMAGERY RECOGNITION COMPARISON AT DIFFERENT NETWORK CONFIGURATIONS USING ALL INDEPENDENT DISTRACTION THEMES ON THE BERLIN-MOTOR IMAGERY

\begin{tabular}{c|ccccc}
\hline Model & BCNN & Em-LSTM & FBCSP & CPMixedNet & GSNN \\
\hline \hline calibration & 0.0153 & 0.0998 & 0.1382 & 0.0030 & $\mathbf{0 . 0 0 3 0}$ \\
clean & 0.0064 & 0.0980 & 0.1589 & 0.0045 & $\mathbf{0 . 0 0 6 6}$ \\
eyes & 0.0098 & 0.0956 & 0.1552 & 0.0028 & $\mathbf{0 . 0 0 2 8}$ \\
flicker & 0.0110 & 0.0908 & 0.1522 & 0.0056 & $\mathbf{0 . 0 0 3 0}$ \\
news & 0.0066 & 0.1016 & 0.1116 & 0.0072 & $\mathbf{0 . 0 1 0 3}$ \\
numbers & 0.0027 & 0.0890 & 0.1570 & 0.0050 & $\mathbf{0 . 0 0 4 6}$ \\
stimulation & 0.0070 & 0.0592 & 0.1473 & 0.0041 & $\mathbf{0 . 0 0 5 4}$ \\
\hline
\end{tabular}

By constructing different blocks, the optimal number of convolutional channels can be filtered out. The accuracy is shown in Table V, in which it can be seen that higher performance can be achieved when four gfNN blocks are applied. It can therefore be concluded that selecting appropriate parameters is conducive to improving classification performance.

TABLE IV

ACCURACY COMPARISON OF THE GSNN WITH DIFFERENT COV-KERNEL TYPES

\begin{tabular}{cccc}
\hline \multicolumn{4}{c}{ Accuracy } \\
\hline Cov-kernel type & GCN & SGC & gfNN \\
\hline \hline calibration & 0.7404 & 0.7784 & 0.7839 \\
clean & 0.7136 & 0.7629 & 0.7884 \\
eyes & 0.7424 & 0.7366 & 0.7953 \\
flicker & 0.7446 & 0.7540 & 0.8076 \\
news & 0.6995 & 0.7546 & 0.7643 \\
numbers & 0.6945 & 0.7211 & 0.7991 \\
stimulation & 0.7481 & 0.7522 & 0.7844 \\
\hline
\end{tabular}

\section{Analysis of Important Brain Regions and Interchannel Structures}

We identified important brain regions of MI based on independent distraction themes. Figure 5 presents a heat map of the diagonal elements in the adjacency matrix we learned, where each subgraph depends on the classification of independent distraction themes. These values were scaled to the $[0,1]$ range for better visualization. Intuitively, diagonal values represent the contribution of each channel to the calculation of the final EEG representation. Figure 5 depicts that there is strong activation in the prefrontal, parietal, and temporal lobes of all frequency bands, suggesting that these areas may be closely related to MI in the brain. Our findings are consistent with existing research. Figure 5 demonstrates the first 17 connections between the channels with the largest edge weight in the adjacency matrix. After A is learnt and sparse selection is completed based on the selection parameter $G$ value, global link-ages 
TABLE V

ACCURACY COMPARISON OF THE GSNN WITH DIFFERENT BLOCK NUMBERS

\begin{tabular}{ccccc}
\hline \multicolumn{5}{c}{ Accuracy } \\
\hline Block number & $\mathbf{2}$ & $\mathbf{3}$ & $\mathbf{4}$ & $\mathbf{5}$ \\
\hline \hline calibration & 0.7078 & 0.7524 & 0.7847 & 0.7971 \\
clean & 0.6049 & 0.7548 & 0.7856 & 0.7606 \\
eyes & 0.6706 & 0.7229 & 0.7972 & 0.7344 \\
flicker & 0.6111 & 0.7043 & 0.8193 & 0.7975 \\
news & 0.6400 & 0.7414 & 0.7672 & 0.7545 \\
numbers & 0.7102 & 0.7210 & 0.7938 & 0.8066 \\
stimulation & 0.7105 & 0.7543 & 0.7819 & 0.7816 \\
\hline
\end{tabular}

remain the strongest, confirming that the relationship between global channels is essential for MI. Additionally, Figure 5(a) shows that the connection between the channel pairs $(\mathrm{C} 3, \mathrm{C} 5)$ is the strongest when imagining the right side at the independent distraction theme, followed by (CCP3, C5) and (CCP1, CP3). The connection between the channel pairs (CCP4, C6) is the strongest when imagining the left side of the task, in the case of the independent distraction theme, followed by $(\mathrm{C} 2, \mathrm{C} 4)$ and $(\mathrm{CCP} 2, \mathrm{CP} 4)$. We also found that the topological connection strength of (F5, FC3) and (F4, FC5) on the prefrontal lobe are related to the distraction on the left and right side imagery, indicating that the relationship between channels in the local frontal region may be important for MI.

\section{Other prospects of the model}

In the process of representation learning with atlas, a better pooling method is needed for learning and selecting important topological features. Because pooling is not a decisive factor, it is difficult to determine whether a global pooled architecture or a hierarchical pooled architecture is beneficial to graph classification in MI. The global pool architecture minimizes information loss, making this architecture perform better than the hierarchical pool architecture on datasets with fewer nodes. However, layering is more effective on datasets with numerous nodes because it can effectively extract useful information from large-scale graphs. Therefore, for the data set of distracted MI, the pooling weight super-parameter setting of the two parts still requires a more precise model.

\section{E. CONCLUSION}

In this study, we trained the sample datasets specific to each subjects at each independent distraction theme. The model adaptively adjusted the subjects' influence at different distraction themes. The problem of noise samples was solved by using the feature standard of regularization and network method in the nodal domain to solve the influence difference of subjects. The sensitivity of the model to different subjects was reduced. Finally, the results showed that pertaining to the task of MI during distraction, our model performances were significantly better than those of the state-of-the-art models. Moreover, the interchannel structures of active brain regions were revealed at different distraction themes, to a certain extent, which provides basic knowledge for additional research. 


\section{CONFLICTS OF INTEREST}

The authors declare that the research was conducted in the absence of any commercial or financial relationships that could be construed as a potential conflict of interest.

\section{REFERENCES}

[1] K. K. Ang, Z. Y. Chin, H. Zhang, and C. Guan, "Filter bank common spatial pattern (fbcsp) in brain-computer interface," Proceedings of the International Joint Conference on Neural Networks, pp. 2390-2397, 2008.

[2] K. P. Thomas, C. T. Lau, A. P. Vinod, C. Guan, and K. K. Ang, "A new discriminative common spatial pattern method for motor imagery brain-computer interfaces," IEEE Transactions on Biomedical Engineering, vol. 56, pp. 2730-2733, 2009.

[3] Q. Novi, C. Guan, T. H. Dat, and P. Xue, "Sub-band common spatial pattern (sbcsp) for brain-computer interface," Proceedings of the 3rd International IEEE EMBS Conference on Neural Engineering, pp. 204-207, 2007.

[4] G. Pfurtscheller, C. Guger, and H. Ramoser, "Eeg-based brain-computer interface using subject-specific spatial filters," Lecture Notes in Computer Science (including subseries Lecture Notes in Artificial Intelligence and Lecture Notes in Bioinformatics), vol. 1607, pp. 248-254,1999.

[5] J. Decety and D. H. Ingvar, "Brain structures participating in mental simulation of motor behavior: A neuropsychological interpretation," Acta Psychologica, vol. 73, pp. 13-34, 21990.

[6] L. M. Alonso-Valerdi, R. A. Salido-Ruiz, and R. A. Ramirez-Mendoza, "Motor imagery based brain-computer interfaces: An emerging technology to rehabilitate motor deficits," Neuropsychologia, vol. 79, pp. 354-363, 122015.

[7] S. Bajaj, A. J. Butler, D. Drake, and M. Dhamala, "Brain effective connectivity during motor-imagery and execution following stroke and rehabilitation," NeuroImage: Clinical, vol. 8, pp. 572-582, 12015.

[8] E. V. Friedrich, R. Scherer, K. Sonnleitner, and C. Neuper, "Impact of auditory distraction on user performance in a brain-computer interface driven by different mental tasks," Clinical Neurophysiology, vol. 122, pp. 2003-2009, 102011.

[9] J. J. Alvarsson, S. Wiens, and M. E. Nilsson, "Stress recovery during exposure to nature sound and environmental noise," International Journal of Environmental Research and Public Health, vol. 7, pp. 1036-1046, 2010.

[10] E. Marx, T. Stephan, A. Nolte, A. Deutschländer, K. C. Seelos, M. Dieterich, and T. Brandt, "Eye closure in darkness animates sensory systems," NeuroImage, vol. 19, pp. 924-934, 72003.

[11] K. Hüfner, T. Stephan, S. Glasauer, R. Kalla, E. Riedel, A. Deutschländer, T. Dera, M. Wiesmann, M. Strupp, and T. Brandt, "Differences in saccade-evoked brain activation patterns with eyes open or eyes closed in complete darkness," Experimental Brain Research, vol. 186, pp. 419-430, 42008.

[12] P. Xu, R. Huang, J. Wang, N. T. V. Dam, T. Xie, Z. Dong, C. Chen, R. Gu, Y. F. Zang, Y. He, J. Fan, and Y. jia Luo, "Different topological organization of human brain functional networks with eyes open versus eyes closed," NeuroImage, vol. 90, pp. 246-255, 42014.

[13] R.Salvador, J.Suckling, M.R. Coleman, J.D. Pickard, D.Menon, and E.Bullmore, "Neurophysiological architecture of functional magnetic resonance images of human brain," Cerebral Cortex, vol. 15, pp. 1332-1342, 92005.

[14] Y. Li, X. R. Zhang, B. Zhang, M. Y. Lei, W. G. Cui, and Y. Z. Guo, "A channel-projection mixed-scale convolutional neural network for motor imagery eeg decoding," IEEE Transactions on Neural Systems and Rehabilitation Engineering, vol. 27, pp. 1170-1180, 62019.

[15] P. Wang, A. Jiang, X. Liu, J. Shang, and L. Zhang, "Lstm-based eeg classification in motor imagery tasks," IEEE Transactions on Neural Systems and Rehabilitation Engineering, vol. 26, pp. 2086-2095, 112018.

[16] R. Li, Q. Wu, J. Liu, Q. Wu, C. Li, and Q. Zhao, "Monitoring depth of anesthesia based on hybrid features and recurrent neural network," Frontiers in Neuroscience, vol. 0, p. 26, 22020.

[17] T. N. Kipf and M. Welling, "Semi-supervised classification with graph convolutional networks," 5th International Conference on Learning Representations, ICLR 2017 - Conference Track Proceedings, 92016.

[18] M. Defferrard, X. Bresson, and P. Vandergheynst, "Convolutional neural networks on graphs with fast localized spectral filtering," Advances in Neural Information Processing Systems, vol. 29, 2016.

[19] P. Veličković, G. Cucurull, A. Casanova, A. Romero, P. Liò, and Y. Bengio, "Graph attention networks," 6th International Conference on Learning Representations, ICLR 2018 - Conference Track Proceedings, 102017.

[20] D. Bahdanau, K. Cho, and Y. Bengio, "Neural machine translation by jointly learning to align and translate," 3rd International Conference on Learning Representations, ICLR 2015 - Conference Track Proceedings, 92014. 
[21] H. NT and T. Maehara, "Revisiting graph neural networks: All we have is low-pass filters," 52019.

[22] A. Gretton, K. M. Borgwardt, M. J. Rasch, A. Smola, B. Schölkopf, and A. S. GRETTON, "A kernel two-sample test bernhard schölkopf," Journal of Machine Learning Research, vol. 13, pp. 723-773, 2012.

[23] M. Ghifary, W. B. Kleijn, M. Zhang, D. Balduzzi, and W. Li, "Deep reconstruction-classification networks for unsupervised domain adaptation," Lecture Notes in Computer Science (including subseries Lecture Notes in Artificial Intelligence and Lecture Notes in Bioinformatics), vol. 9908 LNCS, pp. 597-613, 2016.

[24] Y. Ganin, E. Ustinova, H. Ajakan, P. G. T. journal of machine, and undefined 2016, "Domain-adversarial training of neural networks," jmlrorg.

[25] P. Zhong, D. Wang, and C. Miao, "Eeg-based emotion recognition using regularized graph neural networks," IEEE Transactions on Affective Computing, pp. 1-1, 52020.

[26] S. Brandl, L. Frølich, J. Höhne, K.-R. Müller, W. Samek, and F. Heinrich, "Brain-computer interfacing under distraction: an evaluation study," Journal of Neural Engineering, vol. 13, p. 56012, 2016.

[27] S. Brandl and B. Blankertz, "Motor imagery under distraction - an open access bci dataset," Frontiers in Neuroscience, vol. 0, p. 967, 10 2020.

[28] M. Zhang, Z. Cui, M. Neumann, and Y. Chen, "An end-to-end deep learning architecture for graph classification," Thirty-Second AAAI Conference on Artificial Intelligence 42018.

[29] R. Ying, C. Morris, W. L. Hamilton, J. You, X. Ren, and J. Leskovec, "Hierarchical graph representation learning with differentiable pooling," Advances in Neural Information Processing Systems, vol. 2018-December, pp.4800-4810, 62018.

[30] O. A. Rosso, S. Blanco, J. Yordanova, V. Kolev, A. Figliola, M. Schürmann, and E. B. ar, "Wavelet entropy: a new tool for analysis of short duration brain electrical signals," Journal of Neuroscience Methods, vol. 105, pp. 65-75, 12001.

[31] R. Salvador, J. Suckling, M. R. Coleman, J. D. Pickard, D. Menon, and E. Bullmore, "Neurophysiological architecture of functional magnetic resonance images of human brain," Cerebral Cortex, vol. 15, pp. 1332-1342, 92005.

[32] S. Achard and E. Bullmore, "Efficiency and cost of economical brain functional networks," PLOS Computational Biology, vol. 3, p. e17, 22007.

[33] C. A. A. Cangea, P. Veličkovi 'veličkovi 'c, N. Jovanovi 'cjovanovi 'c, T. Kipf, and P. Liò, "Towards sparse hierarchical graph classifiers," 112018.

[34] X. Glorot and Y. Bengio, "Understanding the difficulty of training deep feedforward neural networks," pp. 249-256, 32010.

[35] O. Shchur, M. Mumme, A. Bojchevski, and S. Günnemann, "Pitfalls of graph neural network evaluation," 112018.

[36] S. Zhao, X. Cui, Y. Fan, C. Zou, and D. Yu, "Binary convolutional neural network for brain computer interfaces," China Semiconductor Technology International Conference 2019, CSTIC 2019, 32019.

[37] T. Hohmann, M. Exner, and N. Schott, "The role of vision and auditory distraction on the temporal congruence between physical execution and motor imagery," Journal of Imagery Research in Sport and Physical Activity, vol. 11, pp. 25-33, 12016.

[38] Y. Liu, W. Su, Z. Li, G. Shi, X. Chu, Y. Kang, and W. Shang, "Motor-imagery-based teleoperation of a dual-arm robot performing manipulation tasks," IEEE Transactions on Cognitive and Developmental Systems, vol. 11, pp. 414-424, 92019.

[39] R. Zhang, Y. Li, Y. Yan, H. Zhang, S. Wu, T. Yu, and Z. Gu, "Control of a wheelchair in an indoor environment based on a brain-computer interface and automated navigation," IEEE Transactions on Neural Systems and Rehabilitation Engineering, vol. 24, pp. 128-139, 12016.

[40] F. Wu, T. Zhang, A. H. de Souza Jr. au2, C. Fifty, T. Yu, and K. Q. Weinberger, "Simplifying graph convolutional networks," 2019. 


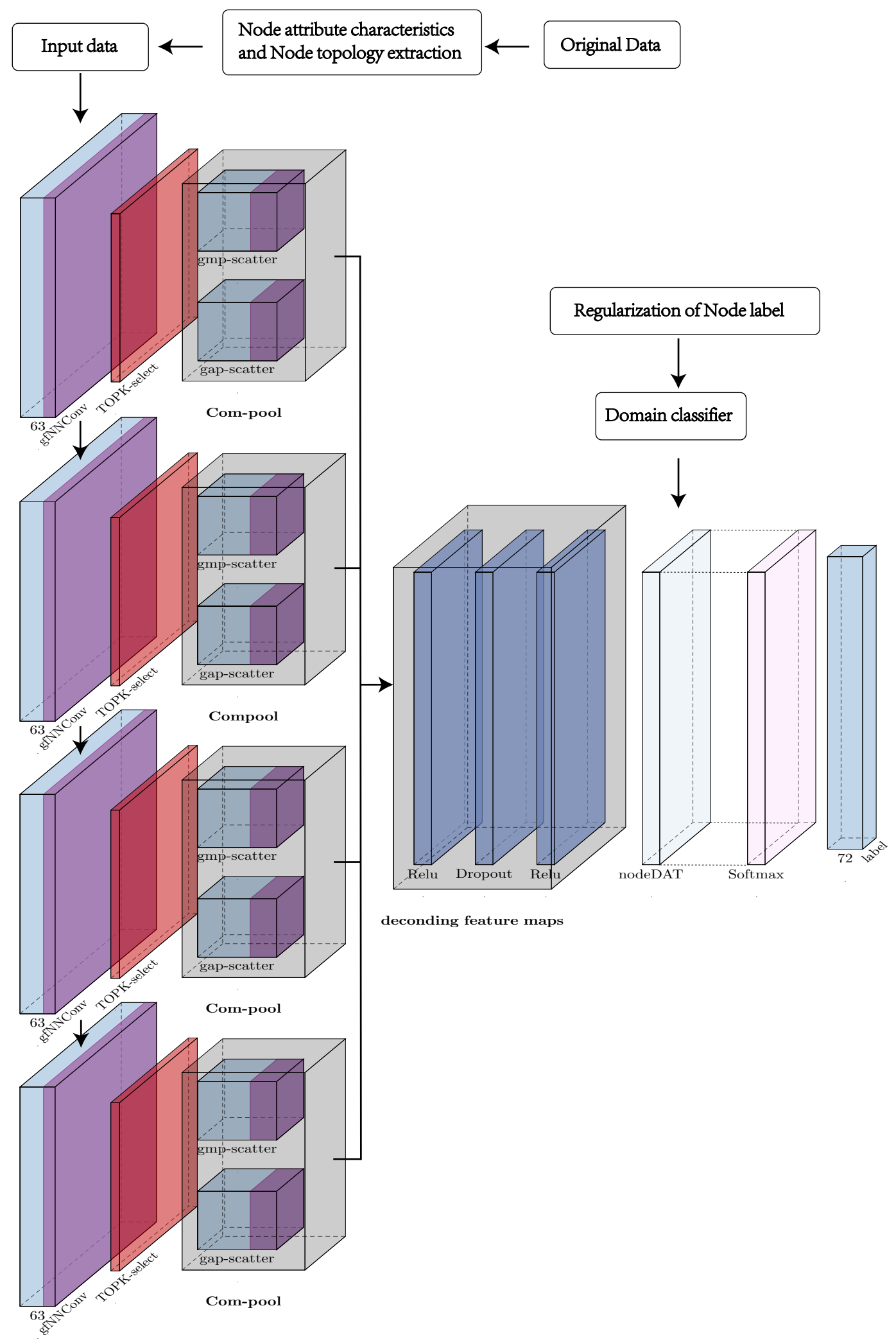

Fig. 3. Architectural design of the proposed graph sequence neural network (GSNN). The structure is a shrinking form, that is, the GSNN network inputs $63 \times 128$ node attribute features, and $63 \times 63$ node connection features, and finally outputs a class label through softmax to evaluate the model. 


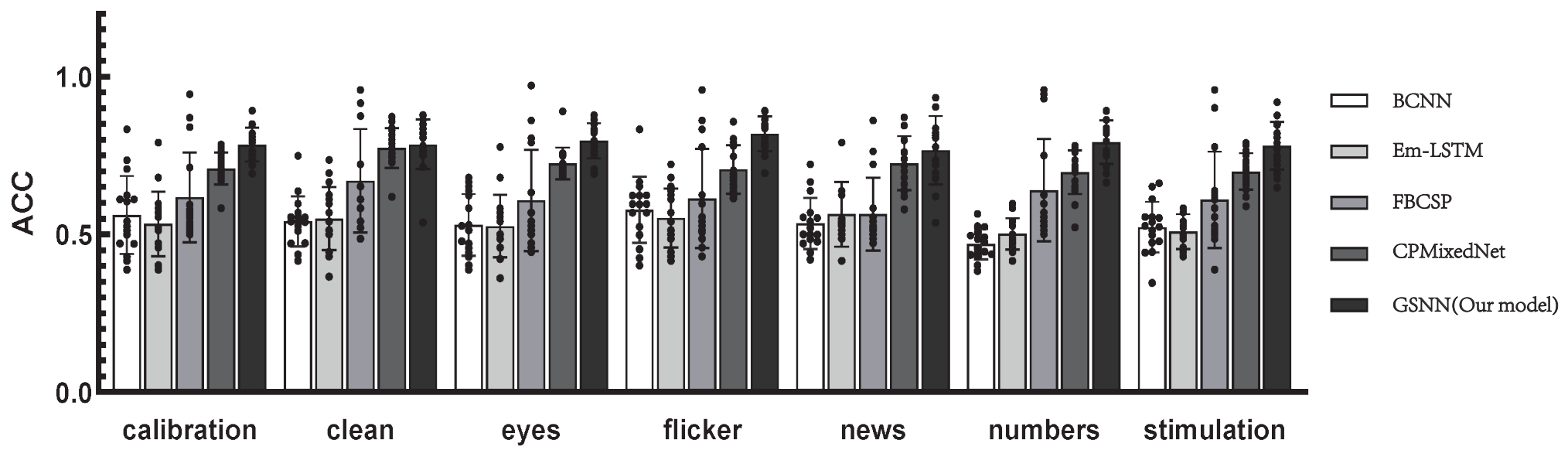

Fig. 4. Results show that our proposed method achieves a performance comparable to the other supervised methods, whereby the recognition accuracies (ACC) of calibration, clean, eye close, flicker, news number, and stimulation are $78.47 \%, 78.56 \%, 79.72 \%, 81.93 \%, 76.72 \%$, $79.38 \%$, and $78.19 \%$, respectively. Each point represents the accuracy of a subject performing a motor imagery task at an independent distraction theme.

(a). Right

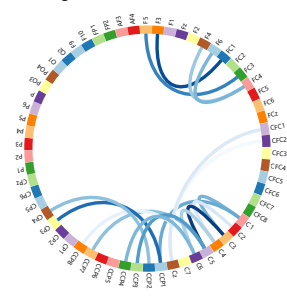

(b). Left

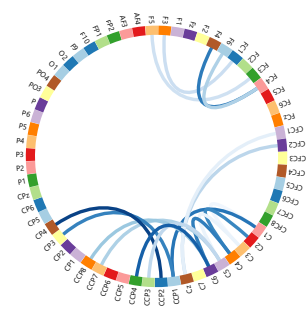

Clean

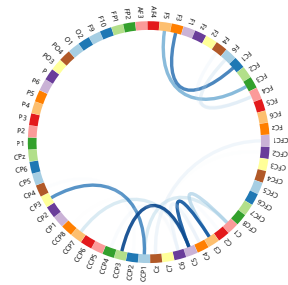

Eyes

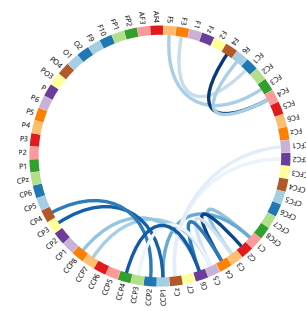

Eyes

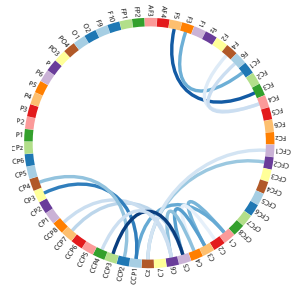

Flicker

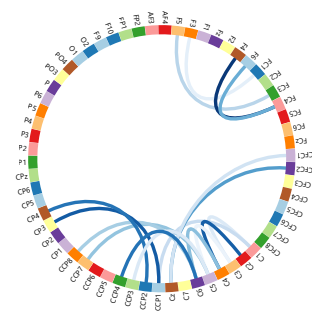

Flicker

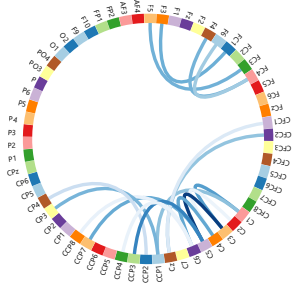

News

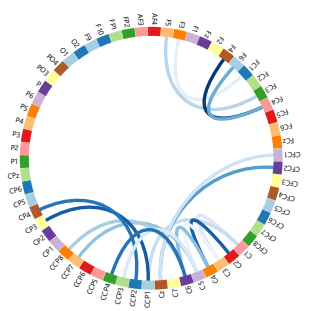

News

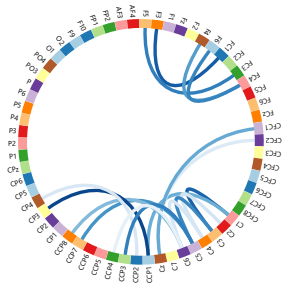

Numbers

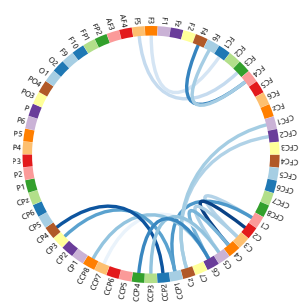

Numbers

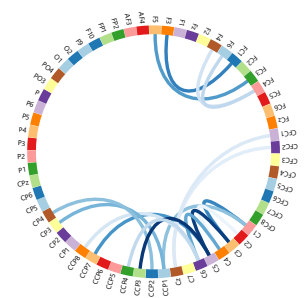

Stimulation

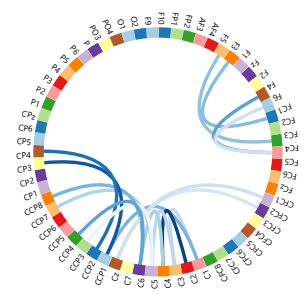

Stimulation

Fig. 5. (a) Top 17 edges between leads in the adjacency matrix A of right imagery, excluding global connections for better clarity. (b) Top 17 edges between leads in the adjacency matrix A of left imagery in subject-dependent classification on the Berlin-motor imagery at the distraction database 